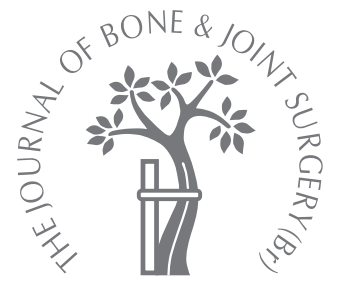

M. Stenning,
S. Drew,
R. Birch

From the Royal

National

Orthopaedic

Hospital, Stanmore, England

\title{
Low-energy arterial injury at the shoulder with progressive or delayed nerve palsy
}

We describe 20 patients, aged between 43 and 88 years, with delayed nerve palsy or deepening of an initial palsy caused by arterial injury from low-energy injuries to the shoulder. The onset of palsy ranged from immediately after the injury to four months later. There was progression in all the patients with an initial partial nerve palsy. Pain was severe in 18 patients, in 16 of whom it presented as neurostenalgia and in two as causalgia. Dislocation of the shoulder or fracture of the proximal humerus occurred in 16 patients. There was soft-tissue crushing in two and prolonged unconsciousness from alcoholic intoxication in another two.

Decompression of the plexus and repair of the arterial injury brought swift relief from pain in all the patients. Nerve recovery was generally good, but less so in neglected cases. The interval from injury to the repair of the vessels ranged from immediately afterwards to 120 days.

Delayed onset of nerve palsy or deepening of a nerve lesion is caused by bleeding and/or impending critical ischaemia and is an overwhelming indication for urgent surgery. There is almost always severe neuropathic pain.

The axillary artery is commonly ruptured in high-energy injuries around the shoulder girdle, which are associated with multiple ruptures of nerves. ${ }^{1}$ However, the artery or its branches are also easily damaged by lowenergy injuries in older people with atheromatous vessels. In a review of 22 cases of arterial injury from such injuries, 16 of the patients were older than 50 years of age. ${ }^{2}$ The consequences of neglect of a late bleed from a false aneurysm were described in a report of an 80year-old woman who suffered a fracture of the humerus. Bleeding re-commenced after four months leading to erosion of the humerus. The diagnosis was not made for two weeks, by which time the upper limb was ischaemic and disarticulation of the shoulder was necessary. ${ }^{3}$

The peripheral nerves have a rich blood supply. The intrinsic systems form a robust longitudinal system within the epi-, peri- and endoneurium. Extrinsic vessels pass to the nerve trunk, branching in the mesoneurium and some of these, such as the superior ulnar collateral vessels, are large enough to sustain free vascularised nerve grafts. The two systems form "separate but extensively interconnected microvascular systems". 4 The robustness of the intrinsic circulation was shown in one patient in whom the flexor muscles of the fore- arm were excised for ischaemia leaving the median nerve nourished by its longitudinal supply over a length of $24 \mathrm{~cm}$. In a second case, the posterior muscles of the thigh were excised for gas gangrene with the sciatic nerve maintained by its longitudinal blood supply over a length of $38 \mathrm{~cm}$. There was useful recovery in both of these nerve trunks. ${ }^{1}$

It is difficult to render a nerve trunk ischaemic. ${ }^{5}$ An example occurred in a case of severe Volkmann's ischaemic contracture for which amputation was done and in which the branches of the median nerve within the palm of the hand were found to be completely infarcted. ${ }^{6}$ In another case, painful ischaemia of the sciatic nerve was relieved by reconstruction of the internal iliac artery. ${ }^{7}$

Clinically, it is difficult to separate the effect on the nerve of pure ischaemia from those associated with compression, traction or distortion. Ischaemia of a nerve is most often caused by compression by tense swollen muscles or by stretching over an expanding haematoma or within the sac of a pulsatile aneurysm or arteriovenous fistula. There are extremes of ischaemia. The transient conduction block of temporary ischaemia was described by Lewis, Pickering and Rothschild ${ }^{8}$ as a centripetal paralysis created by the appli- 
cation around the arm of a cuff inflated to suprasystolic pressure. The large myelinated fibres are affected first; Cfibres and efferent autonomic fibres escape and recovery of all function occurs within a few minutes of release of the cuff. Extreme prolonged ischaemia of the nerve and of the muscles surrounding it leads to infarction of the nerve, effectively a neurotmesis, with destruction not only of the conducting elements but also the myelin of the Schwann cells and the connective tissues. The effect on the nerve is proportional to the duration and the depth of the ischaemia from whatever cause.

The branches of the axillary artery form multiple anastomoses which develop a web-like arcade supplying the thoracic and scapular/humeral areas. The brachial plexus lies in close relation to the axillary artery, within a common fascial sheath. Damage to the artery which may cause only minimal swelling may lead to early compression of the brachial plexus and its components. Barros D'Sa noted that it is sometimes difficult to recognise critical ischaemia after injuries to the axillary artery because of the extensive anastomotic network of collateral vessels around the shoulder. He said "The distal limb may look perfectly viable to the extent that even a pulse may be maintained........ failure to recognise and surgically correct an axillary artery injury may result in an expanding false aneurysm and marked compression of the cords and nerves of the brachial plexus within a limited space". 9 The persistence of peripheral pulses with normal capillary refill may lead the unwary clinician to neglect the underlying arterial injury.

We describe a series of 20 patients in whom the nerves were anatomically intact. Loss of function and pain were indications of critical ischaemia of the nerve, the muscles and, usually, the limb.

\section{Patients and Methods}

Between 1982 and 2002, we treated 152 patients in whom there was rupture of the subclavian or axillary artery from closed injuries to the shoulder girdle. In 132 of these patients the injuries were of the high-energy transfer pattern with multiple ruptures of nerve trunks within the supra- or infraclavicular brachial plexus. The other 20 patients, described here, had either low-energy injuries from either falls in the street or in the home, from softtissue crushing or as the result of a coma.

The mean age was 67 years (43 to 88 ) with 12 patients being older than 60 years of age. They were followed for at least two years from the definitive operation. In one of these patients, the artery had been repaired in the primary hospital before referral. Of the remaining 19, the arterial lesion was repaired in 17 and in the remaining two formal repair of the vessels was not considered to be necessary. In seven patients reduction of the dislocation or internal fixation of the fracture of the humerus was undertaken at the first hospital.

Arterial injury. In most cases, the level of arterial injury was immediately apparent on clinical examination. Arteriogra-

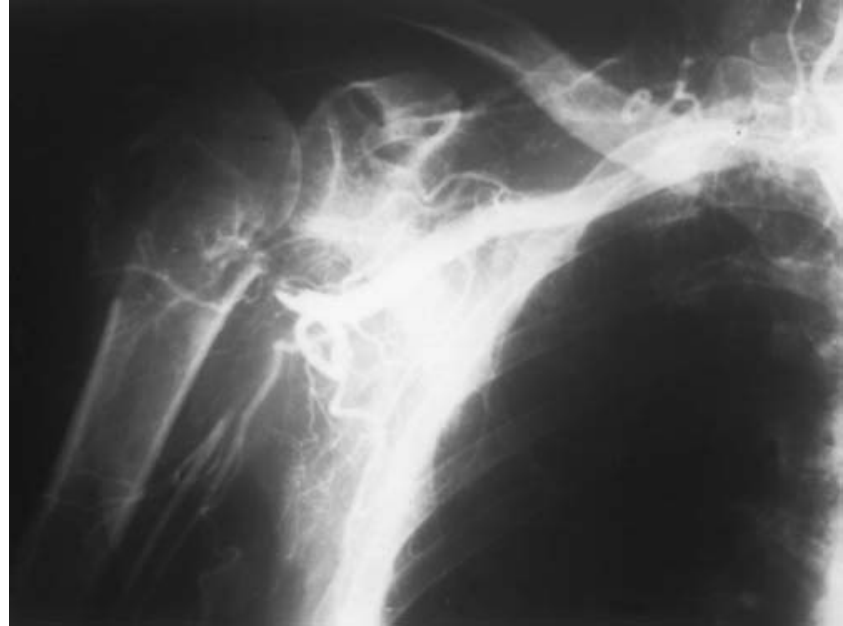

Fig. 1

Arteriogram of the right shoulder of a 66-year-old man showing occlusion of the axillary artery by a fracture of the neck of the humerus.

phy or digital subtraction angiography was performed before operation in seven of the 20 patients (Fig. 1).

The exposure was based on that of Fiolle and Delmas. ${ }^{10}$ In most cases the subclavian artery was isolated and controlled, where it traversed the first rib, using a transverse supraclavicular exposure. Then, through an extended deltopectoral incision, pectoralis major and pectoralis minor were detached from the humerus and from the coracoid process, respectively. It was not necessary to divide the clavicle in any case.

Eleven of the ruptures of the axillary artery were repaired by reversed vein grafts; in one this was undertaken at the primary treating hospital. In two patients with rupture and occlusion of the axillary artery, formal repair of the vessel was considered to be unnecessary at exposure. Of the seven patients with avulsion of branches of the axillary artery, the defect was oversewn in five and repaired by a vein patch in two. Anticoagulants were never used.

Nerve palsy. The infraclavicular part of the brachial plexus was exposed from the origin of the cords to the terminal branches. The nerves were decompressed. Limited external neurolysis was undertaken, preserving the vascular pedicles when possible, and avoiding disturbance to the epineurium. Records of conduction across the lesion and in the distal trunk were made in most cases. ${ }^{11}$ Persisting conduction across the lesion confirmed a good prognosis. Loss of conduction in the distal segments of nerves exposed four or five days after injury showed a degenerative lesion. An epidural catheter was placed adjacent to the cords of the plexus to allow infusion of local anaesthetic for up to 72 hours after operation.

The nerve palsy was defined as a conduction block or a degenerative lesion. In a conduction block the lesion is not complete, usually with preservation of some modalities of 


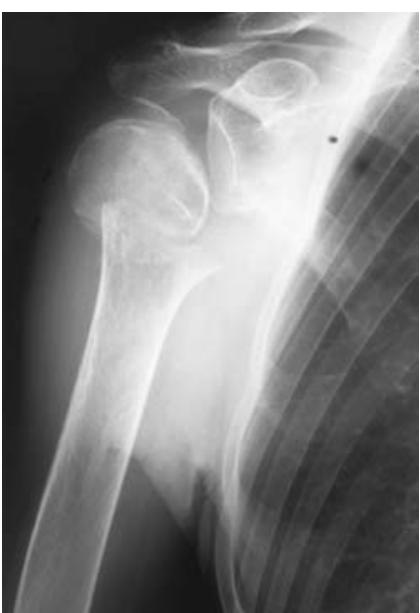

Fig. $2 a$

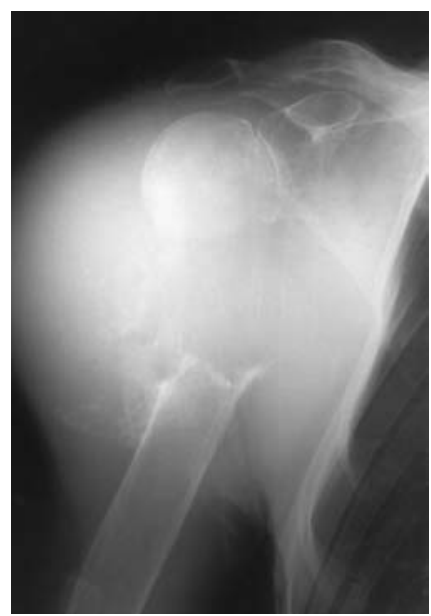

Fig. $2 b$

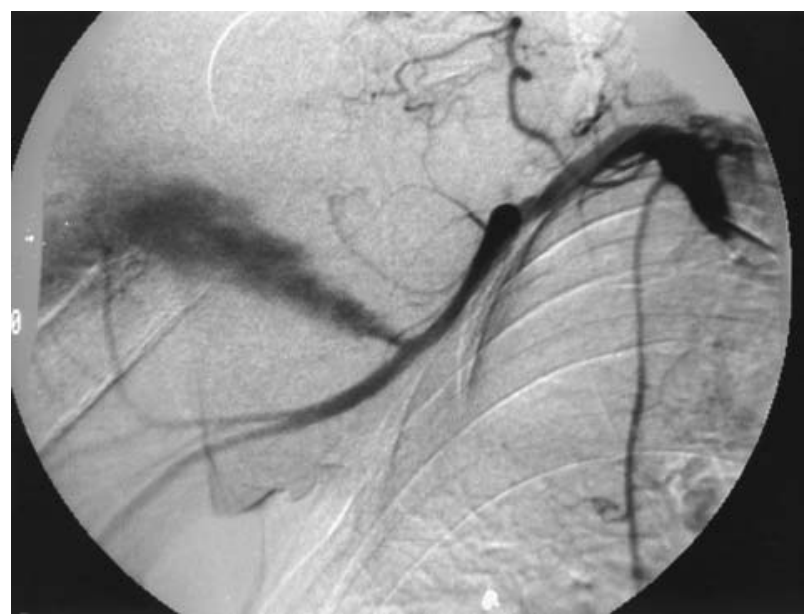

Fig. 2c

Radiograph showing a) a fracture of the right proximal humerus in a 79-year-old woman and b) massive swelling and erosion of the bone 16 weeks later. Figure 2c-Digital subtraction angiography showing bleeding from the axillary artery into a false aneurysm (reproduced with permission from: Birch R, Bonney E, Wynn Parry C, eds. Surgical disorders of the peripheral nerves. Edinburgh: Churchill Livingstone, 1988)

feeling and of sympathetic function. Conduction persists in the nerve distal to the lesion. In a degenerative lesion there is loss of all modalities of function and paralysis of sympathetic post-ganglionic efferent fibres giving sudo- and vasomotor paralysis. Conduction is lost in the distal segment. Recovery of the nerve palsies was classified using the grading of the Medical Research Council. ${ }^{12,13}$

Pain. Two neuropathic pain syndromes were observed, neurostenalgia and causalgia. Neurostenalgia occurs when the nerve, which is anatomically intact, is distorted and trapped, compressed or ischaemic. The pain may be intense throughout the territory of the affected nerve with no clinical evidence of sympathetic overactivity. Removal of the cause is followed, in nearly every case, by relief from the pain and recovery of the nerve, but if the significance of neurostenalgia is ignored and the provocative lesion left untreated, the lesion of the nerve may progress from one of a conduction block to damage of much greater severity. ${ }^{1,14}$

Causalgia, the most severe of the neuropathic pain states, is rare. It was defined by Barnes in $1954 .{ }^{15}$ There is injury to a nerve trunk. The pain is spontaneous and persistent, and usually has a burning quality; it extends beyond the territory of the involved nerve and is invariably aggravated by physical or emotional stimuli. There is mechanical allodynia, intolerance of light stimulation of the skin which would not normally evoke pain, and hyperpathia, the deep burning and cramping pain provoked by palpation of the muscles. Evidence of sympathetic overactivity, of excess sweating and vasomotor disturbance is usual. This syndrome is caused by partial laceration of a trunk of a nerve root in the upper or lower limb. Causalgia in the upper limb after penetrating missile injuries has been related to a false aneurysm or arteriovenous fistula of the adjacent axial artery. Correction of the arterial lesion with repair of the nerve successfully resolves the pain and restores function. ${ }^{15}$

\section{Results}

Arterial injury. The main vessel was ruptured or occluded in 13 patients, and the circumflex, humeral or subscapular branches were avulsed in seven more. There was critical ischaemia in seven limbs. Continuous bleeding was lifethreatening in three patients. Among the secondary consequences of the arterial injury were six massive haematomas, six false aneurysms and three instances of massive secondary bleeding from a false aneurysm. Continuous insidious bleeding continued for six weeks in one patient and eight weeks in another before detection. Massive secondary haemorrhage occurred in one patient at eight weeks from the initial injury, and in two more after 16 weeks (Fig. 2). Repair of the arterial injury ensured adequate peripheral flow in all cases, with restoration of the peripheral pulses and resolution of the oedema. There was no instance of post-ischaemic fibrosis. Fixed deformity within the small joints of the hand required extensive treatment by physical methods in two of the older patients in whom treatment had been delayed.

Nerve palsy. Onset of nerve palsy was evident within six hours in six patients and within 24 hours in five more. It ranged from one or seven days in two limbs, and from eight days to 16 weeks in seven more. In nearly every case the nerve palsy was initially incomplete and there was steady worsening over the ensuing 12 to 24 hours. This deepening in cases of late onset of palsy was diagnostic of a secondary haemorrhage.

Table I summarises the nerves affected and the outcome. Of the 87 nerve palsies, 69 recovered rapidly and were examples of a conduction block (neurapraxia). In 18 the lesion was a deeper one of Wallerian degeneration. Twelve of these went on to full recovery, and four, three median and one ulnar nerve, made useful but incomplete recovery. These 16 favourable degenerative lesions are examples of 
Table I. Palsies by individual trunk and by lesion in 87 nerves

\begin{tabular}{|c|c|c|c|c|c|c|}
\hline & Median & Ulnar & Radial & Musculocutaneous & Circumflex & Total \\
\hline Conduction block (neurapraxia) & 9 & 17 & 18 & 16 & 9 & 69 \\
\hline Favourable degenerative (axonotmesis) & $\begin{array}{l}10 \text { (of which } 3 \text { did } \\
\text { not recover fully) }\end{array}$ & $\begin{array}{l}2 \text { (of which } 1 \text { did } \\
\text { not recover fully) }\end{array}$ & 1 & 1 & 2 & $\begin{array}{l}16 \text { (of which } 4 \text { did } \\
\text { not recover fully) }\end{array}$ \\
\hline Unfavourable degenerative (neurotmesis) & 1 & & & & 1 & 2 \\
\hline Total & 20 & 19 & 19 & 17 & 12 & 87 \\
\hline
\end{tabular}

axonotmesis. In two nerves recovery was very poor. These were degenerative lesions of unfavourable prognosis (neurotmesis). The median nerve was most commonly affected probably because of the particularly close relationship between its trunk and the axial artery. The median nerve was regularly found to be involved in peri-arterial fibrosis, within an expanding haematoma or incorporated into the wall of a false aneurysm. One median nerve, which was greatly distorted by incorporation within the wall of a false aneurysm, did not recover. Neurotmesis of one circumflex nerve was caused by encirclement within a suture at the first operation.

Pain. The onset of pain preceded recognition of the nerve palsy in five patients. In another 13 it coincided with the nerve lesion and worsened as that lesion deepened. Five patients attended a pain clinic. The use of drugs and, in two cases, of a sympathetic block, was invariably futile. Neuropathic pain presented as neurostenalgia in 16 patients, and as causalgia in two more. In two patients neuropathic pain was never present.

The corrective operation was performed within 48 hours of the onset of nerve palsy and pain in ten patients, and at seven days in another. In nine patients the interval between the onset of the nerve lesion and operation ranged from eight to 120 days, and in five of these exceeded 28 days. There was early and permanent relief from pain in all patients, and in most this was apparent on the first day after operation. There were no serious complications and no wound infections.

\section{Discussion}

In some patients an expectant approach was adopted to the arterial injury once it had been recognised. The presence of peripheral pulses and apparently adequate capillary return in the skin was taken as evidence that the ischaemia within the limb was not critical. Eastcott, Blaisdell and Silver, ${ }^{16}$ defined the "imminent condition of severe irreversible acute ischaemia". The danger signs were waxy pallor, severe pain and numbness, with the hand or foot being much colder than the opposite limb, and muscle weakness, paralysis, tenderness or rigidity being present. In all of our 20 patients there was a deepening neural defect, and 18 had worsening pain, irrespective of the presence or absence of peripheral pulses and the state of circulation to the skin.

The diagnosis of arterial spasm was made in four patients before they were referred. Barros $\mathrm{d}^{\prime} \mathrm{Sa}^{17}$ wrote that "true spasm is rare and represents a local myogenic re- action of the arterial wall... it is probably accompanied by endothelial damage and some contusion of the adventitia. A diagnosis of spasm is both presumptive and dangerous in that it leads to inactivity in the presence of a limb threatening arterial injury". We agree that the concept of arterial spasm should be largely disregarded in clinical practice although one of our patients, a 58 -year-old man, does represent a true instance of arterial spasm. Distal flow was restored once the artery had been extricated from the fracture of the humerus in which it had been entrapped for seven days.

Arterial injury was implicated in all of these patients by the delayed onset of nerve palsy or deepening of a nerve palsy inflicted at the time of injury, by worsening neuropathic pain which usually presented as neurostenalgia, by local swelling or bruising within the axilla and in seven by clear signs of ischaemia of the limb as a whole.

Fractures or dislocations of the shoulder are extremely painful, but the pain should be eased greatly by the reduction of the dislocation or stabilisation of the fracture. Persistent or worsening pain after primary treatment, particularly when it is associated with deepening of nerve palsy, strongly indicates that the bleeding is persisting and that the brachial plexus is becoming compromised by an expanding haematoma or false aneurysm. Unchecked, this bleeding may threaten the viability of the limb. Late secondary haemorrhage from a false aneurysm is life-threatening.

No benefits in any form have been received or will be received from a commercial party related directly or indirectly to the subject of this article.

\section{References}

1. Birch R, Bonney G. Compound nerve injuries: the vascular lesion. In: Birch R, Bonney G, Wynn Parry CB, eds. Surgical disorders of the peripheral nerves. First ed. London: Churchill Livingstone, 1998:125-34.

2. Gates JD, Knox JB. Axillary artery injuries secondary to anterior dislocation of the shoulder. J Trauma 1995;39:581-3.

3. Syed AA, Williams HR. Shoulder disarticulation: a sequel of vascular injury secondary to a proximal humeral fracture. Injury 2002;33:771-4.

4. Lundborg G. Vascular systems. In: Nerve injury and repair. Edinburgh: Churchill Livingstone, 1988:32-63.

5. McManis PG, Low PA, Lagerlund TD. Microenvironment of nerve: blood flow and ischaemia. In: Dyck PJ, Thomas PK, Griffin JW, Low PA, Poduslo JF, eds. Peripheral neuropathy. Third ed. Philadelphia: W.B. Saunders Co. 1993:433-40.

6. Seddon HJ. Neurovascular injuries. In: Surgical disorders of peripheral nerves. 2nd Edition. Edinburgh, etc: Churchill Livingstone, 1975:89-111.

7. Lamerton AJ, Bannister R, Withrington R, Seifert MH, Eastcott HHG. “Claudication" of the sciatic nerve. Brit Med J (Clin Res Ed) 1983;286:1785-6.

8. Lewis T, Pickering GW, Rothschild P. Centripetal paralysis arising out of arrested bloodflow to the limb: including notes on a form of tingling. Heart 1931;16:1-32.

9. Barros D'Sa. Arterial injuries. In: Eastcott HHG, ed. Arterial surgery. Third ed. London: Churchill Livingstone, 1992:355-413. 
10. Fiolle J, Delmas J. In: Cumston CG (trans ed). The surgical exposure of the deep seated blood vessels. London: Heinemann, 1921:61-7.

11. Birch R, Bonney G. Operating on peripheral nerves. In: Birch R, Bonney G, Wynn Parry CB, eds. Surgical disorders of the peripheral nerves. First ed. London: Churchill Livingstone, 1998:87-106.

12. Birch R, Bonney G. Results. In: Birch R, Bonney G, Wynn Parry C, eds. Surgical disorders of the peripheral nerves. First ed. London: Churchill Livingstone, 1998:235-42.

13. Seddon HJ. Results of repair of nerves. In: Surgical disorders of peripheral nerves. Second ed. Edinburgh: Churchill Livingstone, 1975:303-14.
14. Stewart M, Birch R. Penetrating missile injuries of the brachial plexus. J Bone Joint Surg [Br] 2001;83-B:517-24.

15. Barnes R. Causalgia: a review of 48 cases. In: Seddon HJ, ed. Peripheral nerve injuries by the Nerve Injuries Committee of the Medical Research Council. Medical Research Council Special Report series 282. Ed. London: Her Majesty's Stationery Office, 1954:156-85.

16. Eastcott HHG, Blaisdell FW, Silver D. Acute ischaemia. In: Eastcott HHG, ed. Arterial surgery. Third ed. Edinburgh: Churchill Livingstone, 1992:31-54.

17. Barros d'Sa. Arterial injuries. In: Eastcott HHG, ed. Arterial surgery. Third ed. Edinburgh: Churchill Livingstone, 1992:355-413, 363-4. 